\title{
Progenitor expansion in apc mutants is mediated by Jak/Stat signaling
}

Junji Lin, Xu Wang and Richard I Dorsky

\begin{abstract}
Background: Mutations in APC, a negative regulator of the Wnt/B-catenin pathway, can cause cancer as well as profound developmental defects. In both cases, affected cells adopt a proliferative progenitor state and fail to differentiate. While the upregulation of some target genes of Wnt/ß-catenin signaling has been shown to mediate these phenotypes in individual tissues, it is unclear whether a common mechanism underlies the defects in APC mutants.

Results: Here we show that stat3, a known oncogene and a target of B-catenin in multiple tissues, is upregulated in apc mutant zebrafish embryos. We further demonstrate that Jak/Stat signaling is necessary for the increased level of proliferation and neural progenitor gene expression observed in apc mutants.
\end{abstract}

Conclusions: Together, our data suggest that the regulation of Jak/Stat signaling may represent a conserved mechanism explaining the expansion of undifferentiated cells downstream of APC mutations.

Keywords: Wnt, APC, Stat3, progenitor, zebrafish

\section{Background}

Apc loss causes progenitor expansion in development and disease

The Wnt/ß-catenin signaling pathway acts to maintain the undifferentiated progenitor state in multiple epithelial tissues, and overactivation of this pathway is a major contributor to cancer. The tumor suppressor APC normally functions to inhibit Wnt/ß-catenin signaling, and $A P C$ mutations are oncogenic in tissues such as the colorectal epithelium [1]. During normal embryonic development, Wnt and APC activities are balanced to allow both progenitor cell expansion and differentiation of postmitotic derivatives. Zebrafish embryos homozygous for $a p c$ mutations exhibit mispatterning and failure of differentiation in multiple tissues including the central nervous system (CNS) [2,3]. Furthermore, in the CNS of other vertebrates, loss of APC function specifically leads to arrest in the neural progenitor state [4]. Despite a clear picture of the cellular phenotypes following loss of $\mathrm{APC}$, the molecular pathways underlying CNS progenitor cell expansion are largely unknown. These pathways

\footnotetext{
* Correspondence: richard.dorsky@neuro.utah.edu

Department of Neurobiology and Anatomy, University of Utah School of Medicine, Salt Lake City, UT 84132, USA
}

may represent good candidates for mediators of oncogenesis in other epithelial cells.

\section{Transcriptional targets of Wnt signaling mediate $A P C$ mutant phenotypes}

The main downstream output of Wnt/B-catenin signaling is the transcriptional regulation of target genes, mediated by Lef/Tcf family members. Typically, these targets are repressed by Lef/Tcf factors in the absence of Wnt signaling, and following Wnt activation $ß$-catenin translocates to the nucleus where it binds to Lef/Tcf proteins and acts as a co-activator. The identification of Wnt/ß-catenin transcriptional targets has thus been a major focus of investigation in past studies of the pathway's role in development and disease. Some identified target genes have been shown to be common targets in both normal embryos and the oncogenic state. For example, mitf is a direct target of Lef1 during melanocyte specification, and also plays an important role in melanoma progression downstream of Wnt pathway hyperactivation $[5,6]$. Similarly, Wnt targets such as ascl 2 and $\lg 5$ may function in both intestinal epithelium homeostasis as well as colon cancer $[7,8]$. 


\section{Stat3 functions synergistically with Wnt signaling in cancer}

Like Wnt signaling, the Jak/Stat pathway has been shown to mediate proliferation and tumor growth in cancer. In particular, constitutive Stat3 activity is associated with malignancy in colon cancer [9], the primary carcinoma caused by APC mutations. A previous study showed that Wnt signaling can stimulate Stat3 activity during early zebrafish development [10], but the mechanism underlying this activation was not characterized. One potential mechanism of regulation has been suggested by a study in esophageal carcinoma, where Stat 3 was shown to be a transcriptional target of $ß$-catenin via Tcf4 [11]. Intriguingly, Stat3 has also been suggested to be a target of Wnt signaling in ES cells [12], suggesting that this pathway may represent a developmentally important mechanism. However, the regulatory relationship between Wnt signaling and Stat3 activation has not been explored in vivo in untransformed tissue.

Here we demonstrate that stat3 is a direct transcriptional target of $\mathrm{Wnt} / \mathrm{B}$-catenin signaling in developing zebrafish embryos. We show that increased stat3 expression in apc mutants correlates with increased proliferation and failure of neuronal differentiation in the developing hypothalamus. Conditional inhibition of Jak/ Stat signaling rescues proliferation defects as well as ectopic expression of progenitor markers, but not the general activation of Wnt targets or the complete process of neurogenesis. Together, these data indicate a specific function for Jak/Stat activation in mediating neural progenitor expansion downstream of APC mutations, and suggest a conserved role for this pathway in development and disease.

\section{Results and Discussion}

\section{stat3 is a direct target of the Wnt pathway via Lef1}

We have previously shown that Wnt signaling, mediated by the transcriptional effector Lef1, is required for hypothalamic neurogenesis in the zebrafish brain [13]. To identify transcriptional targets of the Wnt pathway, we performed ChIP-seq analysis using a Lef1 antibody. Immunoprecipitation was performed using chromatin from whole 36 hours post-fertilization (hpf) embryos, corresponding with a time of high lef1 expression in the hypothalamus. After deep sequencing of precipitated chromatin, we observed high enrichment of the stat3 promoter region compared to total input as well as chromatin from lef1 deletion mutant embryos. The genomic sequence identified by ChIP-seq (Figure 1A) contains several putative Lef/Tcf consensus binding sites (Figure 1B), and we confirmed the direct interaction with Lef1 using ChIP followed by quantitative PCR (Figure 1C).

We next tested whether the endogenous expression of stat3 in the zebrafish embryo depends on Wnt-mediated transcription. We used a transgenic inducible repressor of Lef/Tcf target genes ( $h s: \Delta T c f)$ to globally inhibit pathway activity in vivo. $28 \mathrm{hpf}$ embryos were heat shocked for one hour, allowed to recover until $36 \mathrm{hpf}$, and then processed for in situ hybridization. We observed a qualitative decrease in stat3 expression throughout embryos expressing $\Delta \mathrm{Tcf}$, including in the hypothalamus (Figure $1 \mathrm{D}, \mathrm{E})$. Together, these results suggest that stat3 is a direct transcriptional target of the Wnt pathway.

\section{stat3 expression and Stat3 phosphorylation are increased in apc mutants}

Previous studies have reported multiple developmental defects in the CNS of apc mutant zebrafish embryos, including axon pathfinding errors [14], loss of normal brain patterning [3], and expansion of the putative retinal stem cell zone [2]. An additional striking phenotype that we observed in mutant embryos was a dramatic increase in proliferating cells particularly in the hypothalamus, accompanied by a dramatic decrease in differentiated neurons (Figure 2A). An earlier study identified stat3 as a marker that was increased in apc mutant embryos in the putative retinal stem cell zone and the hypothalamus [2]. We examined stat 3 expression throughout the apc mutant embryo and observed a qualitative increase in mRNA levels, with specific enrichment in known CNS progenitor zones including the hypothalamus (Figure 2B). Quantitative PCR analysis of apc mutant embryos showed an increase in the level of stat 3 mRNA of $5.34 \pm$ .09 fold (s.d., $\mathrm{n}=3, \mathrm{p}<0.05$ by student's t-test) compared to wild-type siblings. We also found a qualitative increase in pStat3 immunostaining in the apc mutant hypothalamus compared to control embryos (Figure 2B), suggesting that stat 3 mRNA levels may normally limit the signaling output of this pathway. Based on the known roles of Stat3 function in progenitor cell maintenance, these results raised the possibility that increased Jak/Stat signaling might underlie some of the progenitor differentiation defects present in the apc mutant brain.

\section{Increased proliferation in apc mutants can be rescued by blocking Jak/Stat signaling}

In other tissues, $A P C$ mutations and Stat3 hyperactivation can both lead to increased cell proliferation. To quantify the proliferative increase in apc mutant zebrafish, we performed short-pulse (1 hour) BrdU labeling in wild-type and mutant embryos. At $36 \mathrm{hpf}$, significantly more cells within the developing hypothalamus of apc mutant embryos incorporated BrdU than in wild-type siblings (Figure 2C,D). These data are consistent with an increased number of progenitor cells in the CNS of apc mutants compared to wild-type embryos.

We next tested whether inhibition of Jak/Stat activity could reverse the increased proliferation found in apc 


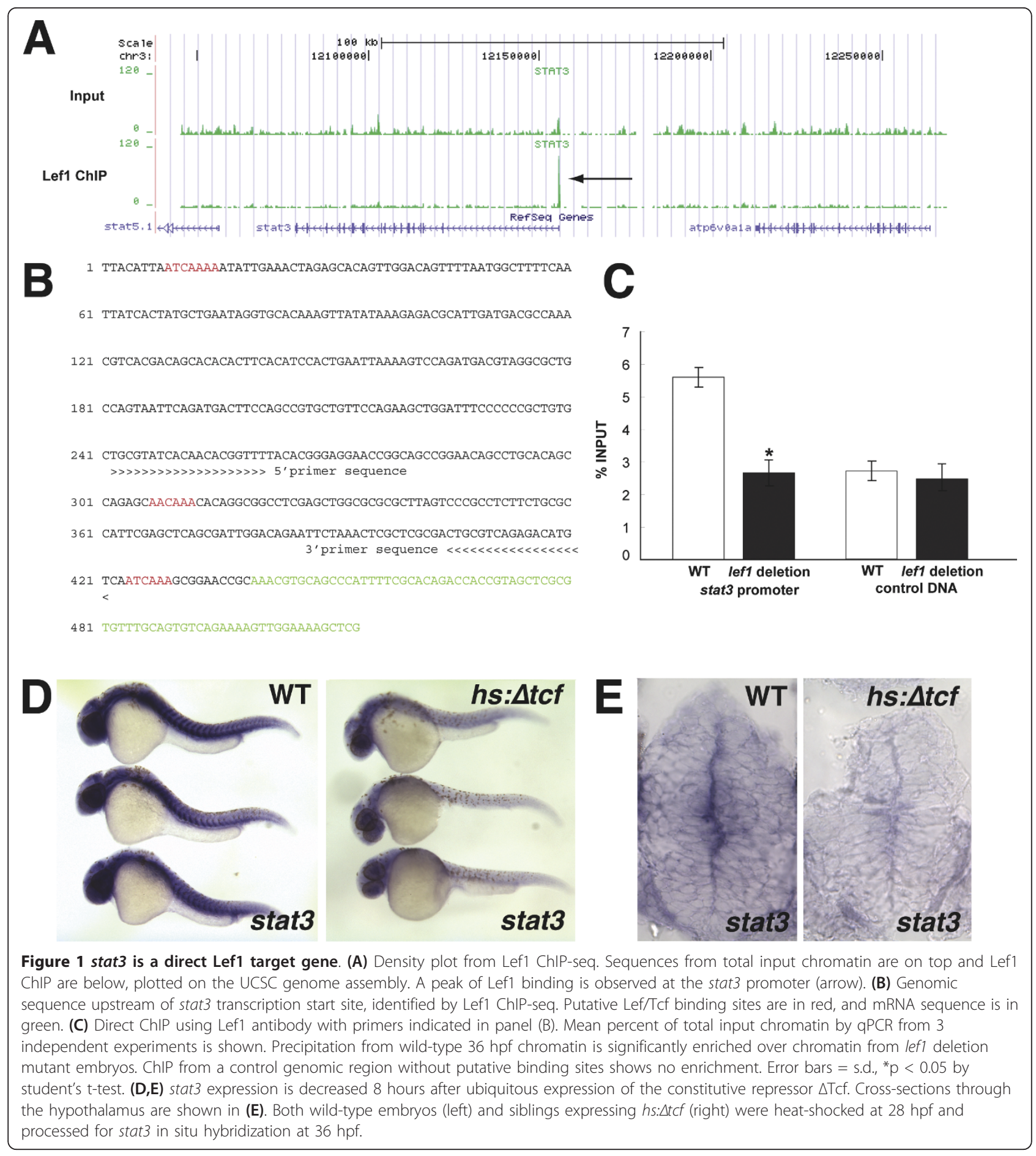

mutants. To block Jak/Stat signaling, we used the Jak2 inhibitor AG-490, which has been demonstrated to prevent Stat3 phosphorylation in many other experimental systems including zebrafish [15] and allowed us to bypass early developmental defects resulting from stat 3 knockdown. When wild-type embryos were incubated in $40 \mu \mathrm{m}$ AG-490 from 24-36 hpf, we did not observe a significant change in the BrdU labeling index compared to untreated controls (Figure 2C,D). In contrast, AG490 incubation completely reversed the increase in proliferation observed in apc mutant embryos, restoring the BrdU labeling index to wild-type levels (Figure 2C,D). Together, these data indicate that Jak/Stat signaling is required for increased proliferation in apc mutant 


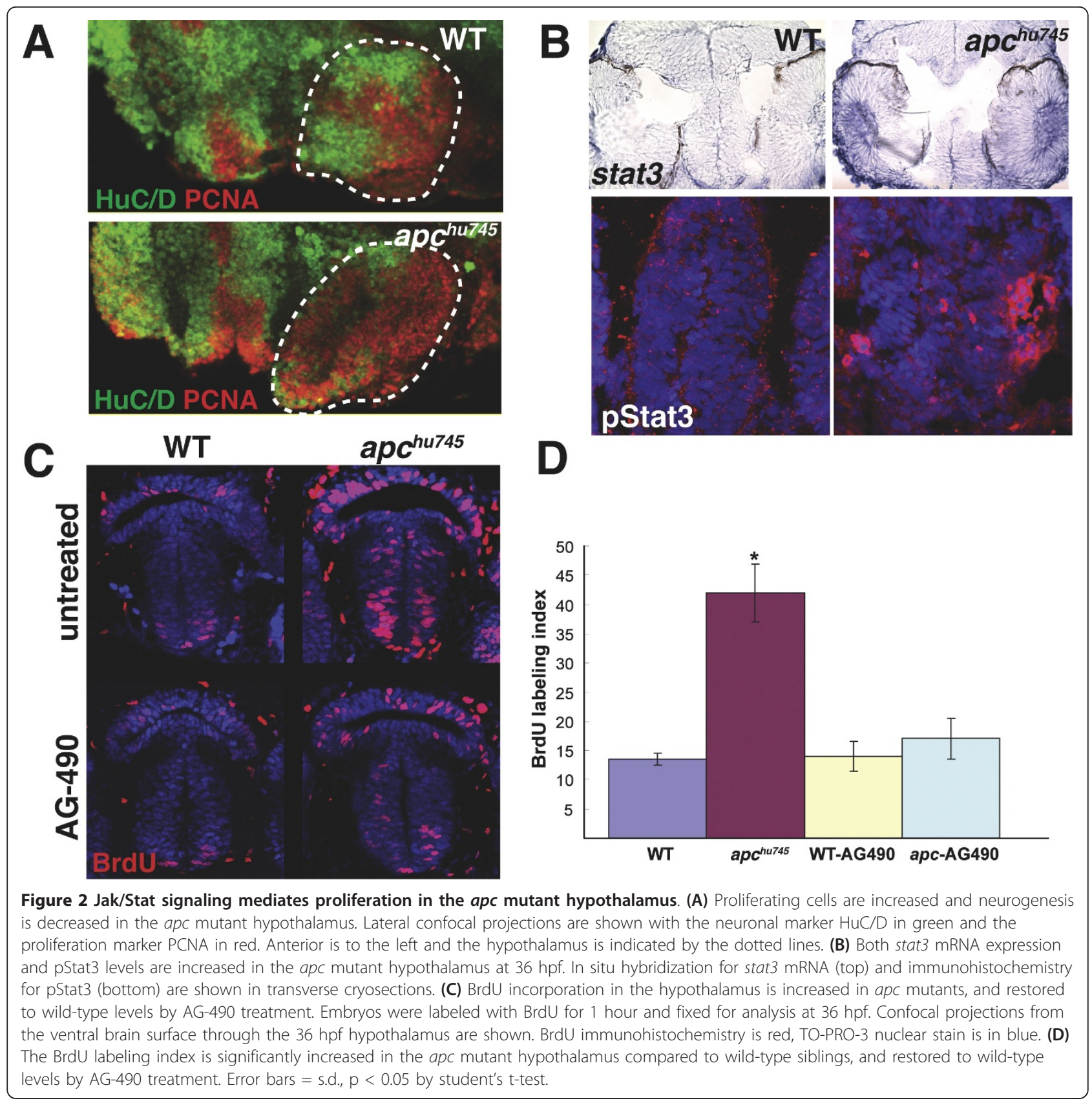

brains. Our observations of increased stat3 mRNA expression in apc mutants suggest that Stat3 levels may be limiting in the developing brain, and that regulation by the Wnt pathway may control the ability of Jak/Stat signaling to drive cell proliferation.

\section{Increased progenitor marker expression in apc mutants requires Jak/Stat activity}

Because proliferation is closely linked to the progenitor cell phenotype in the developing CNS, we wanted to determine whether other markers of neural progenitors were also increased in apc mutants and whether this increase depends on Jak/Stat activity. We first examined the expression of $a s c l 1 b$, which encodes a proneural bHLH transcription factor essential for neurogenesis. Using in situ hybridization, we found that ascl1b mRNA levels were qualitatively increased in the $a p c$ mutant hypothalamus at $36 \mathrm{hpf}$ (Figure 3A). Incubation in $40 \mu \mathrm{M}$ AG-490 from 24-36 hpf was able to eliminate this increase and restore $a s c l 1 b$ expression to wild-type levels in apc mutants (Figure 3A), suggesting that increased proneural gene expression is mediated by Jak/Stat activity. 


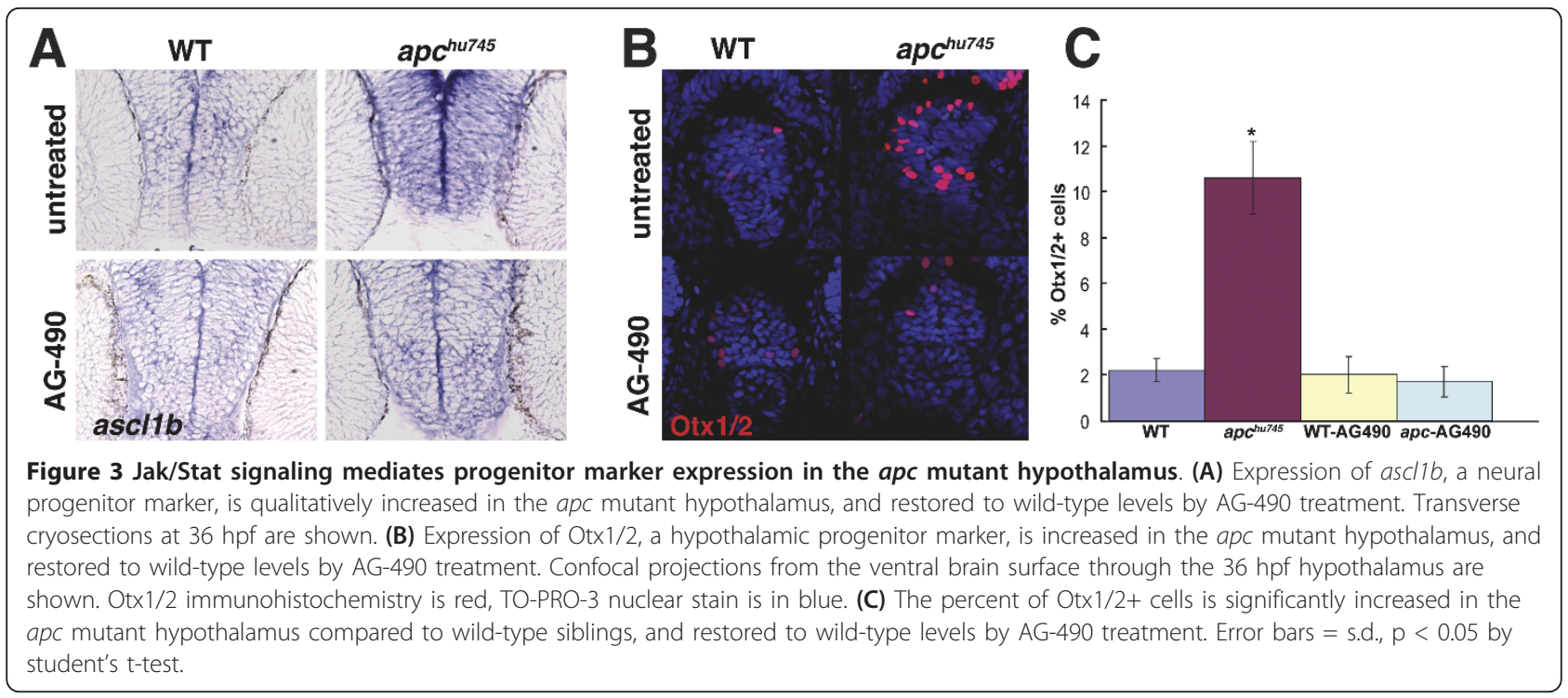

In the zebrafish retina, otx 1 expression marks the putative stem cell zone of the ciliary margin, and is expanded in apc mutants [2]. Otx1 and Otx2 are also expressed in the developing vertebrate hypothalamus and label neural progenitors in the zebrafish hypothalamus. We observed increased otx 1 mRNA expression in the hypothalamus of apc mutants (not shown), and to provide a more quantitative measurement, we examined the number of cells labeled with an antibody that recognizes both Otx1 and Otx2. Within the hypothalamus, $a p c$ mutants showed a significant increase in Otx1/2positive cells at $36 \mathrm{hpf}$ (Figure $3 \mathrm{~B}, \mathrm{C}$ ), and this increase was rescued to wild-type levels by AG-490 incubation (Figure 3B,C). These data suggest that cells may be arrested in an Otx-positive progenitor state following $a p c$ inactivation, and that $\mathrm{Jak} / \mathrm{Stat}$ function mediates this arrest.

\section{Inhibition of Jak/Stat activity is not sufficient to rescue neurogenesis in apc mutants}

While Jak/Stat activity is required for the expansion of CNS progenitor characteristics downstream of apc inactivation and stat 3 transcription, we hypothesized that this pathway is not likely to mediate all outputs of Wnt activation. Indeed, when we examined the expression of the Wnt target gene axin2, we observed a strong increase in mRNA expression that was not rescued by AG-490 incubation (Figure 4A). This result indicates that many transcriptional targets of $\mathrm{Wnt} / \mathrm{B}$-catenin signaling are likely to be independent of Jak/Stat activity, and that these targets may act in parallel pathways. Furthermore, while AG-490 incubation could rescue increases in proliferation and progenitor gene expression, it was insufficient to restore neurogenesis in $a p c$ mutants. The loss of HuC/D expression observed in the hypothalamus was still seen in embryos after incubation in AG-490 (Figure 4B), suggesting that neural progenitors were still unable to differentiate into neurons. Therefore, other Stat3-independent targets of APC must be important for regulating the full program of differentiation. These could possibly include Wnt-independent APC targets, as has been demonstrated previously in other studies [16].

\section{Conclusions}

Here we have shown that stat 3 is a direct transcriptional target of Wnt signaling in the developing embryo, and that Jak/Stat signaling mediates the expansion and maintenance of CNS progenitor characteristics downstream of Wnt hyperactivation in apc mutants. Together, our data suggest that transcriptional regulation of stat 3 may represent a general mechanism linking Wnt pathway overactivation to the expansion of undifferentiated cells in the disease state.

At higher doses of AG-490, we were able to completely eliminate both proliferation and progenitor marker expression in wild-type embryos (not shown). Combined with the endogenous expression pattern of stat3, and the fact that $\Delta$ Tcf can repress stat 3 in wild-type embryos, this suggests that a Wnt/Stat3 pathway may also play an important role in normal CNS development.

\section{Methods}

\section{Zebrafish maintenance and embryo culture}

Embryos were obtained from natural spawning of wildtype (AB*), $T g(h s p 70 l: t c f 3-G F P)^{w 26}, D f(L G 01: l e f 1, m s x b)^{x 8}$, and $a p c^{h u 745}$ mutant zebrafish and were staged 


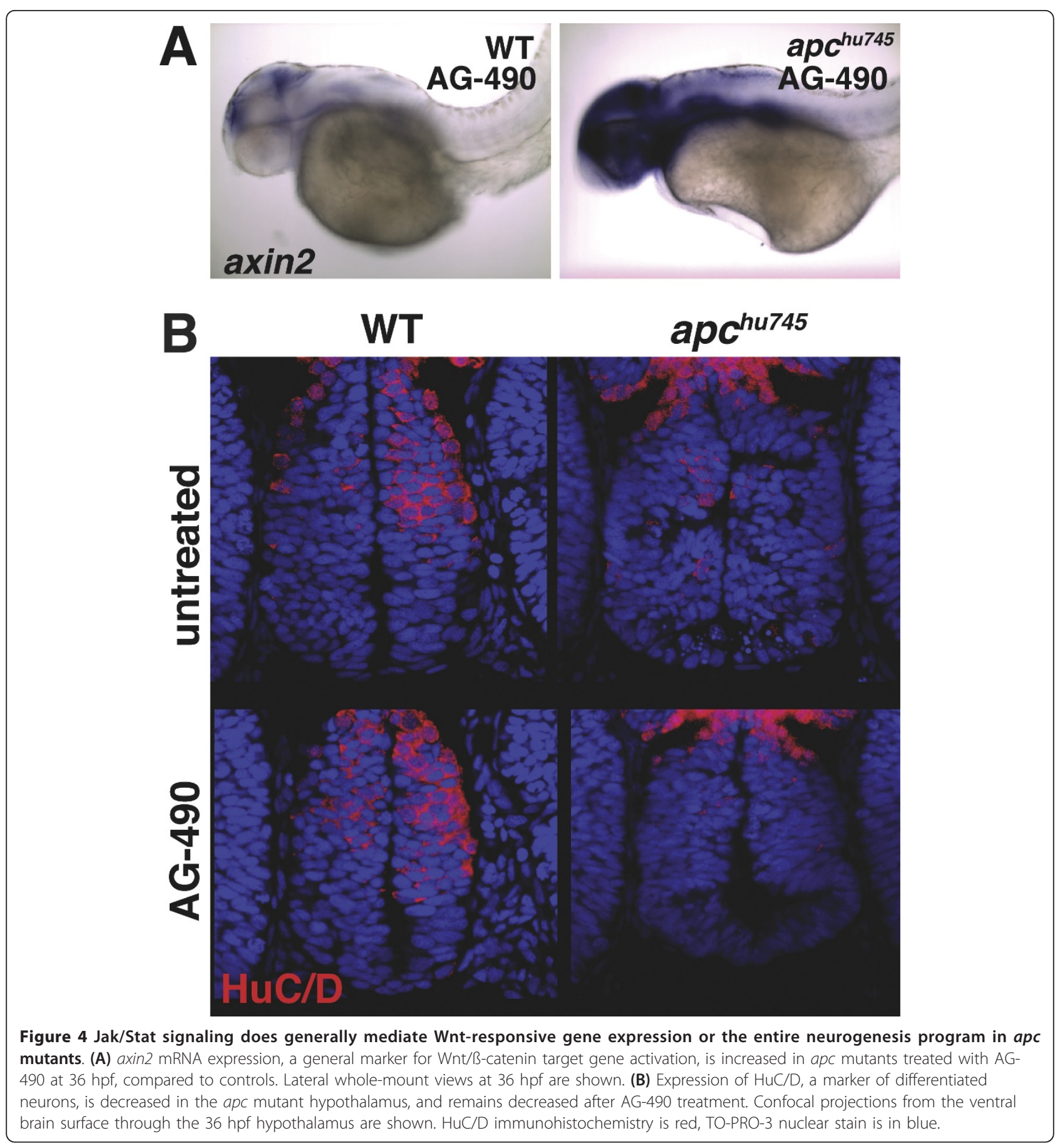

according to Kimmel et al., [17]. lef1 deletion and apc mutant embryos were identified by morphology and $h s$ : $\Delta t c f$ embryos were identified by expression of a GFP fusion protein. All embryos were raised at $28.5^{\circ} \mathrm{C}$ and fixed in $4 \%$ PFA for analysis. $28 \mathrm{hpf} h s: \Delta t c f$ embryos were heat shocked for 1 hour at $37^{\circ} \mathrm{C}$, then allowed to recover at $28.5^{\circ} \mathrm{C}$ until $36 \mathrm{hpf}$. To block Jak/Stat signaling, embryos were treated with 40 uM AG-490 (Enzo) beginning at $24 \mathrm{hpf}$. For BrdU labeling, $35 \mathrm{hpf}$ embryos were incubated in $10 \mathrm{mM}$ BrdU in 15\% DMSO for 30 minutes on ice, washed and allowed to recover for 1 hour at $28.5^{\circ} \mathrm{C}$ before fixation.

\section{ChIP and qPCR}

ChIP analysis was performed as described previously [18] with the following modifications. One hundred 
embryos at $36 \mathrm{hpf}$ were dechorionated and fixed in 1\% PFA in PBS for 15 minutes at room temperature, and then lysed in cell lysis buffer [10 mM Tris ( $\mathrm{pH} 8.1), 10$ $\mathrm{mM} \mathrm{NaCl}, 0.5 \% \mathrm{NP}-40$, and protease inhibitors] and nuclear lysis buffer [50 mM Tris- $\mathrm{Cl}(\mathrm{pH} 8.1), 10 \mathrm{mM}$ EDTA, 1\% SDS and proteinase inhibitors] by pipetting. For each immunoprecipitation, 5 ug of anti-Lef1 antibody [13] was conjugated to $30 \mathrm{ul}$ Dynabeads (Invitrogen) prior to applying nuclear extract. A detailed protocol is posted at: https://wiki.zfin.org/display/prot/ ZFIN+Protocol+Wiki. Precipitated DNA fragments were purified and submitted for Illumina sequencing at the University of Utah HSC Core Facility and sequences were mapped to zebrafish genome (assembly zv7).

For qPCR analysis of ChIP fragments, total input chromatin and Lef1 immunoprecipitated chromatin from wild-type and $D f(L G 01: l e f 1, m s x b)^{x 8}$ mutant siblings was used. For qPCR analysis of stat3 mRNA levels, total RNA was isolated from $42 \mathrm{hpf}$ wild-type and $a p c^{h u 745}$ mutants using an RNAeasy extraction kit (Qiagen) followed by DNase treatment. cDNA was synthesized by SuperScript II reverse transcriptase (Invitrogen), and stat3 levels were normalized to beta actin cDNA. Quantitative real-time PCR was performed at the University of Utah HSC Core Facility.

Primers used for stat3 ChIP qPCR are: 5'-TGCGTATCACAACACGGTTT-3' 5'-ACATGTCTCTGACGCAGTCG-3' Primers used for stat3 cDNA qPCR are: 5'-CCGACTGGAAGAGGAGACAG-3' 5'GCTGGACGGTGCTGAATAAT-3'

\section{In situ hybridization}

Whole mount in situ hybridization was performed as described previously [13]. Probes for stat3 [19] and otx 1 [20] were obtained from T. Piotrowski. Probes for $a s c l 1 b$ and axin 2 were synthesized in our laboratory. Following staining, whole embryos were mounted in $80 \%$ glycerol and imaged on a dissecting microscope, or embedded in plastic, sectioned, and imaged on a compound microscope.

\section{Immunohistochemistry}

For BrdU and PCNA detection, fixed embryos were incubated for 1 hour in $2 \mathrm{~N} \mathrm{HCl}$. Immunostaining was performed as described previously [21]. Antibodies were obtained from the following sources: anti-BrdU (AbD Serotec, 1:500), anti-HuC/D (Molecular Probes, 1:500), anti-OTX1/2 (Chemicon, 1:500), anti-PCNA (Sigma, 1:1000), anti-pStat3 (Tyr708, MBL, 1:1000), and secondary antibodies conjugated to Alexa Fluor 647 (Invitrogen). Following immunohistochemistry, embryos were counterstained with TO-PRO-3 (Invitrogen), and whole brains were dissected for imaging. Embryos were mounted in Fluoromount-G (Southern Biotech), and confocal images were acquired using an Olympus FV1000 microscope.

\section{Acknowledgements and Funding \\ Grant Sponsor: NIH (NINDS); R21NS055138}

\section{Authors' contributions}

J.L. conducted all experiments except the PCNA analysis of apc mutants, qPCR for stat3, and pStat3 staining, which were performed by X.W. R.I.D. provided oversight for the entire study and wrote the manuscript. All authors read and approved the final manuscript.

Received: 2 September 2011 Accepted: 2 December 2011 Published: 2 December 2011

\section{References}

1. Phelps RA, Broadbent TJ, Stafforini DM, Jones DA: New perspectives on APC control of cell fate and proliferation in colorectal cancer. Cell Cycle 2009, 8(16):2549-2556.

2. Stephens WZ, Senecal M, Nguyen M, Piotrowski T: Loss of adenomatous polyposis coli (apc) results in an expanded ciliary marginal zone in the zebrafish eye. Dev Dyn 2010, 239(7):2066-2077.

3. Paridaen JT, Danesin C, Elas AT, van de Water S, Houart C, Zivkovic D: Apc1 is required for maintenance of local brain organizers and dorsal midbrain survival. Dev Biol 2009, 331(2):101-112.

4. Imura T, Wang X, Noda T, Sofroniew MV, Fushiki S: Adenomatous polyposis coli is essential for both neuronal differentiation and maintenance of adult neural stem cells in subventricular zone and hippocampus. Stem Cells 2010, 28(11):2053-2064.

5. Dorsky RI, Raible DW, Moon RT: Direct regulation of nacre, a zebrafish MITF homolog required for pigment cell formation, by the Wnt pathway. Genes Dev 2000, 14(2):158-162.

6. Widlund HR, Horstmann MA, Price ER, Cui J, Lessnick SL, Wu M, He X, Fisher DE: Beta-catenin-induced melanoma growth requires the downstream target Microphthalmia-associated transcription factor. J Cell Biol 2002, 158(6):1079-1087.

7. Haegebarth A, Clevers $\mathrm{H}$ : Wnt signaling, lgr5, and stem cells in the intestine and skin. Am J Pathol 2009, 174(3):715-721.

8. Jubb AM, Chalasani S, Frantz GD, Smits R, Grabsch HI, Kavi V, Maughan NJ, Hillan KJ, Quirke P, Koeppen H: Achaete-scute like 2 (ascl2) is a target of Wnt signalling and is upregulated in intestinal neoplasia. Oncogene 2006, 25(24):3445-3457.

9. Klampfer $\mathrm{L}$ : The role of signal transducers and activators of transcription in colon cancer. Front Biosci 2008, 13:2888-2899.

10. Yamashita S, Miyagi C, Carmany-Rampey A, Shimizu T, Fujii R, Schier AF, Hirano T: Stat3 Controls Cell Movements during Zebrafish Gastrulation. Dev Cell 2002, 2(3):363-375.

11. Yan S, Zhou C, Zhang W, Zhang G, Zhao X, Yang S, Wang Y, Lu N, Zhu H, $\mathrm{Xu} \mathrm{N}$ : beta-Catenin/TCF pathway upregulates STAT3 expression in human esophageal squamous cell carcinoma. Cancer Lett 2008, 271(1):85-97.

12. Hao J, Li TG, Qi X, Zhao DF, Zhao GQ: WNT/beta-catenin pathway upregulates Stat3 and converges on LIF to prevent differentiation of mouse embryonic stem cells. Dev Biol 2006, 290(1):81-91.

13. Lee JE, Wu SF, Goering LM, Dorsky Rl: Canonical Wnt signaling through Lef1 is required for hypothalamic neurogenesis. Development 2006, 133(22):4451-4461

14. Paridaen JT, Danesin C, Elas AT, van de Water S, Houart C, Zivkovic D: Apc1-mediated antagonism of Wnt/beta-catenin signaling is required for retino-tectal pathfinding in the zebrafish. Zebrafish 2009, 6(1):41-47.

15. Ma AC, Ward AC, Liang R, Leung AY: The role of jak2a in zebrafish hematopoiesis. Blood 2007, 110(6):1824-1830.

16. Nadauld LD, Phelps R, Moore BC, Eisinger A, Sandoval IT, Chidester S, Peterson PW, Manos EJ, Sklow B, Burt RW, et al: Adenomatous polyposis coli control of $\mathrm{C}$-terminal binding protein-1 stability regulates expression of intestinal retinol dehydrogenases. J Biol Chem 2006, 281(49):37828-37835.

17. Kimmel CB, Ballard WW, Kimmel SR, Ullmann B, Schilling TF: Stages of embryonic development of the zebrafish. Dev Dyn 1995, 203(3):253-310. 
18. Weinmann AS, Bartley SM, Zhang T, Zhang MQ, Farnham PJ: Use of chromatin immunoprecipitation to clone novel E2F target promoters. Mol Cell Biol 2001, 21(20):6820-6832.

19. Oates AC, Wollberg P, Pratt SJ, Paw BH, Johnson SL, Ho RK, Postlethwait JH, Zon LI, Wilks AF: Zebrafish stat3 is expressed in restricted tissues during embryogenesis and stat1 rescues cytokine signaling in a STAT1-deficient human cell line. Dev Dyn 1999, 215(4):352-370.

20. Li Y, Allende ML, Finkelstein R, Weinberg ES: Expression of two zebrafish orthodenticle-related genes in the embryonic brain. Mech Dev 1994, 48(3):229-244.

21. Gribble SL, Kim HS, Bonner J, Wang X, Dorsky RI: Tcf3 inhibits spinal cord neurogenesis by regulating sox4a expression. Development 2009, 136(5):781-789.

doi:10.1186/1471-213X-11-73

Cite this article as: Lin et al:: Progenitor expansion in apc mutants is mediated by Jak/Stat signaling. BMC Developmental Biology 2011 11:73.

\section{Submit your next manuscript to BioMed Central} and take full advantage of:

- Convenient online submission

- Thorough peer review

- No space constraints or color figure charges

- Immediate publication on acceptance

- Inclusion in PubMed, CAS, Scopus and Google Scholar

- Research which is freely available for redistribution

Submit your manuscript at www.biomedcentral.com/submit 